\title{
The effect of sex and age on the comorbidity burden of OSA: an observational analysis from a large nationwide US health claims database
}

\author{
Babak Mokhlesi ${ }^{1}$, Sandra A. Ham² and David Gozal ${ }^{3}$ \\ Affiliations: 'Sleep Disorders Center and the Section of Pulmonary and Critical Care Medicine, Dept of Medicine, \\ The University of Chicago, Chicago, IL, USA. ${ }^{2}$ Center for Health and the Social Sciences, The University of \\ Chicago, Chicago, IL, USA. ${ }^{3}$ Sections of Pediatric Sleep Medicine and Pulmonology, Dept of Pediatrics, \\ Pritzker School of Medicine, Biological Sciences Division, The University of Chicago, Chicago, IL, USA.
}

Correspondence: Babak Mokhlesi, Section of Pulmonary and Critical Care, Sleep Disorders Center, The University of Chicago, 5841 S. Maryland Ave, MC6076/Room M630, Chicago, IL 60637-1470, USA.

E-mail: bmokhlesamedicine.bsd.uchicago.edu

ABSTRACT Obstructive sleep apnoea (OSA) is a highly prevalent condition but studies exploring the burden of OSA-associated comorbidities have been limited by small sample sizes with underrepresentation of women.

We queried the Truven Health MarketScan Research Databases 2003-2012, which is a collection of health insurance claims for working adults and retirees with employer-sponsored health insurance. Adults with a diagnostic code for OSA with at least 12 months of follow-up from the index date of OSA diagnosis were compared to a matched random sample. Comorbidities were assessed using International Classification of Diseases, Ninth Edition, codes. A logistic regression model was constructed to test the independent association between OSA and comorbidities.

Our cohort included 1704905 patients with OSA and 1704417 matched controls. All comorbidities were significantly more prevalent in OSA patients. Type 2 diabetes and ischaemic heart disease were more prevalent in men but hypertension and depression were more prevalent in women with OSA. In contrast, the sex differences in the prevalence of congestive heart failure, arrhythmias and stroke were less pronounced. The prevalence of comorbidities increased with age but the effect of age varied based on the specific comorbidity. The divergence between OSA and controls was more pronounced after the sixth decade of life for most cardiovascular diseases (i.e. heart failure, ischaemic heart disease, stroke and arrhythmias), while depression exhibited an opposite trend. In a fully adjusted model, the odds of all comorbidities were significantly increased in OSA patients.

In a large, nationally representative sample of working and retired people, OSA is strongly associated with significant comorbidities in both men and women with unique sex differences emerging.

@ERSpublications

OSA is associated with comorbidities in both men and women with a unique pattern of sex and age differences http://ow.ly/V4Rg6

For editorial comment see Eur Respir J 2016; 47: 1047-1049 [DOI: 10.1183/13993003.00060-2016].

This article has supplementary material available from erj.ersjournals.com

Received: Sept 292015 | Accepted after revision: Nov 142015 | First published online: Jan 212016

Support statement: This study was supported in part by the Herbert T. Abelson Chair in Pediatrics to D. Gozal. Funding information for this article has been deposited with FundRef.

Conflict of interest: Disclosures can be found alongside the online version of this article at erj.ersjournals.com

Copyright OERS 2016 


\section{Introduction}

The prevalence of obstructive sleep apnoea (OSA) has been increasing in parallel with the obesity epidemic. Earlier population-based studies reported a prevalence of moderate to severe OSA of $4-7 \%$ in women and $9-14 \%$ in men [1-5]. A more recent study that applied current diagnostic definitions used in clinical sleep laboratories reported a substantially higher prevalence of OSA. In this population-based study from Switzerland, $23 \%$ of women and $49 \%$ of men were found to have moderate to severe OSA [6].

OSA is a clinically relevant chronic condition characterised by recurrent episodes of complete (apnoea) or partial (hypopnea) obstruction of the upper airway causing intermittent hypoxaemia and hypercapnia, cortical microarousals, increased oxidative stress, and sleep fragmentation [1,5]. These adverse effects of OSA are important mediators of cardiovascular, neurocognitive and metabolic risk [7-14]. OSA has been independently associated with type 2 diabetes [14], hypertension [15, 16], coronary heart disease, stroke and cardiovascular mortality [17-21]. Moreover, OSA is a well-recognized risk factor for motor vehicle and work-related accidents, and when untreated, it has been associated with overall increased health morbidity, and cognitive and mood deficits impairing work efficiency and productivity [22, 23].

Prior studies exploring healthcare expenditures and overall burden of comorbidities associated with OSA have included relatively small, single-centre clinical cohorts subject to selection and referral bias [24-28]. There is a paucity of nationally representative cohorts exploring the burden of comorbidities associated with OSA. Jennum and co-workers [29, 30] explored the Danish National Patient Registry, and reported a significantly elevated comorbidity burden and healthcare resource utilisation in patients with OSA compared to controls. However, the generalisability of the findings may be limited, given that there were only 19438 patients with a diagnosis of OSA during an 8-year period in this administrative database. Moreover, women were underrepresented in this cohort. A more recent study from Veterans Administration hospitals and clinics in the USA reported a significant association between OSA and cardiovascular comorbidities in 21 674 patients with untreated OSA [31]. This study was limited by the fact that $96 \%$ of patients with OSA were men. Similarly, a data mining approach on $>60000$ individuals with OSA uncovered a distinct occurrence peak around 55-59 years of age, and multiple comorbid conditions involving the cardiovascular, endocrine/metabolic and respiratory systems, along with increased use of healthcare services [32].

Our primary objective was to delineate more accurately the burden of comorbidities associated with a diagnosis of OSA in a very large, nationwide health insurance database for working adults and retirees with employer-sponsored health insurance coverage in the USA. We also aimed to explore sex differences as well as the potential effect of age in the burden of comorbidities associated with OSA.

\section{Methods}

Data source

The Truven Health MarketScan Research Databases are a collection of health insurance claims for working adults and retirees with employer-sponsored health insurance. For the current project, we used the MarketScan Commercial Claims and Encounters database constructed from paid claims for employee-sponsored health insurance from 2003 to 2012, representing 17 million enrolees in 2003 to 57 million enrolees in 2012. In this study, we used the inpatient service, outpatient service, inpatient admission, facility header and enrolment tables. Within these tables, we used insurance claim records from January 1, 2003, to December 31, 2012.

The study was exempt from ethical review given that the database is de-identified and publically available.

\section{Patient selection}

Between January 2003 and December 2012, there were 77.8 million individuals aged 30-94 years in the Truven Marketscan database. We chose to enrol patients starting at age 30 years based on previously published population-based studies that also enrolled individuals that were 30 years and older $[1,6]$. We queried the database for patients with International Classification of Diseases, Ninth Edition - Clinical Manifestations (ICD-9-CM) diagnosis codes for OSA (table 1). A total of 5166379 million patients had a diagnosis of OSA. We excluded $67 \%$ of patients for not having insurance enrolment for 1 year prior to and 1 year following the date of OSA diagnosis. Therefore, a total of 1704905 OSA patients were included in the analysis.

A matched random sample was selected from enrolees of the same sex within the same 5-year age group and state of residence $(n=1704417)$. Potential matches had at least 24 months of enrolment and did not have any ICD-9-CM codes for inpatient or outpatient diagnoses of OSA, charges for polysomnograms or out-of-centre sleep testing, or charges for continuous positive airway pressure at any time during their enrolment in MarketScan. We did not exclude enrolees who did not have any contact with the healthcare system (i.e. no inpatient or outpatient claims). For example, if an individual had no claims for either OSA or any comorbidity, they could have been selected as a control. 
TABLE 1 Sample characteristics for patients diagnosed with obstructive sleep apnoea (OSA) and matched random samples

\begin{tabular}{lccc} 
& Diagnosed with OSA & Matched random sample & All patients combined \\
\hline Total & $1704905(100)$ & $1704417(100)$ & $3409322(100)$ \\
Men & $855441(50.18)$ & $855321(50.18)$ & $1710762(50.18)$ \\
$30-39$ years & $137109(8.04)$ & $137109(8.04)$ & $274218(8.04)$ \\
$40-49$ years & $237421(13.93)$ & $237421(13.93)$ & $474842(13.93)$ \\
$50-59$ years & $276659(16.23)$ & $276659(16.23)$ & $553318(16.23)$ \\
$60-69$ years & $141296(8.29)$ & $141296(8.29)$ & $282592(8.29)$ \\
$70-79$ years & $46400(2.72)$ & $46400(2.72)$ & $92800(2.72)$ \\
$>79$ years & $16556(0.97)$ & $16436(0.96)$ & $32992(0.97)$ \\
Women & $849464(49.82)$ & $849096(49.82)$ & $1698560(49.82)$ \\
$30-39$ years & $135896(7.97)$ & $135896(7.97)$ & $271792(7.97)$ \\
$40-49$ years & $232535(13.64)$ & $232535(13.64)$ & $465070(13.64)$ \\
$50-59$ years & $283513(16.63)$ & $283513(16.63)$ & $567026(16.63)$ \\
$60-69$ years & $126532(7.42)$ & $126532(7.42)$ & $253064(7.42)$ \\
$70-79$ years & $46682(2.74)$ & $46682(2.74)$ & $93364(2.74)$ \\
$>79$ years & $24306(1.43)$ & $23938(1.40)$ & $48244(1.42)$ \\
\hline
\end{tabular}

Data are presented as $\mathrm{n}(\%)$.

\section{Covariates}

Age in years was ascertained on the date of index OSA diagnosis or the age 1 year into the enrolment period for the matched random sample. Age was classified into 10-year age groups from 30 to 79 years, and >79-94 years. Comorbidities were assessed using the inpatient and outpatient service, inpatient admission, and facility header tables. For OSA cases, we aimed to minimise the risk of reporting bias (i.e. new diagnosis of OSA leading the clinicians to diagnose additional comorbidities) by querying for diagnosis of comorbidities for the 6 months prior to the index diagnosis of OSA being established. In other words, all comorbidities reported in our analyses were established before the diagnosis of OSA. Comorbidity data was extracted from months 7 to 12 of enrolment for the matched random sample. Patients were considered to have comorbidity if an associated ICD-9-CM code was found at least once in the data tables. Using Elixhauser comorbidity classifications, we constructed six comorbidity categories (table 2 and supplementary table S1). In addition, we classified individuals as having morbid obesity, hypertension, type 2 diabetes, ischaemic heart disease, congestive heart failure, stroke, arrhythmias or depression using ICD-9-CM codes in table S2. To illustrate sex differences in the prevalence of comorbidities between patients with OSA and matched controls, age was classified into 5-year groups.

\section{Statistical analysis}

We summarised the distribution of demographics and comorbidities for OSA cases, and the matched random sample using descriptive analyses. We performed Chi-squared tests for bivariate analyses of

\begin{tabular}{|c|c|c|c|}
\hline & Diagnosed with OSA & Matched random sample & p-value \\
\hline Total & $1704905(100)$ & $1704417(100)$ & \\
\hline Cardiovascular diseases & $643130(37.73)$ & 316593 (18.57) & $<0.0001$ \\
\hline Metabolic diseases & 332631 (19.52) & 129327 (7.59) & $<0.0001$ \\
\hline Chronic pulmonary disease & $183441(10.76)$ & 59302 (3.48) & $<0.0001$ \\
\hline Renal failure & $28295(1.66)$ & $9587(0.56)$ & $<0.0001$ \\
\hline Liver disease & 32971 (1.93) & $13154(0.77)$ & $<0.0001$ \\
\hline Psychological conditions & $223224(13.1)$ & $60685(3.56)$ & $<0.0001$ \\
\hline
\end{tabular}

Data are presented as $\mathrm{n}(\%)$ unless otherwise stated. Cardiovascular diseases included categories for congestive heart failure, cardiac arrhythmia, valvular disease, pulmonary circulation disorders and hypertension. Metabolic diseases included categories for diabetes and obesity. Psychological conditions included categories for drug and alcohol abuse, depression, and psychoses. 
categorical variables. We used two-sided tests, with p-values $<0.05$ considered to be statistically significant. Prevalence of OSA-related comorbidities by age group, sex and group (i.e. OSA or control) were determined. Logistic regression models tested the odds ratios of having OSA-related comorbidities after adjusting for age group and sex. All statistical analyses were performed with SAS 9.3 (SAS Institute, Cary, NC, USA).

\section{Results}

Our analytic cohort included 1704905 patients carrying an ICD-9-CM code diagnosis for OSA. Our controls included 1704417 without a diagnosis of OSA matched for age, sex and state of residence (table 1). The comorbidity characteristics based on Elixhauser comorbidity categories are shown in table 2. We also used ICD-9-CM codes as described in table S2 to characterise better the prevalence of OSA-specific comorbidities (table 3). All comorbidities explored were significantly more prevalent in patients carrying a diagnosis of OSA when compared to matched controls.

Figure 1 illustrates the effect of age and sex on the prevalence of comorbidities in patients with OSA and matched controls. All comorbidities were more prevalent in patients with OSA regardless of sex when compared to matched controls, especially with increasing age. However, the prevalence of type 2 diabetes and ischaemic heart disease was higher in men with OSA compared to women with OSA. In contrast, hypertension and depression were more prevalent in women with OSA. Sex differences in the prevalence of arrhythmias and stroke in patients with OSA were much less pronounced. Type 2 diabetes, hypertension and depression were significantly more prevalent in patients with OSA compared to matched controls at all age groups.

A logistic regression model was constructed to estimate the odds of having comorbidities after adjusting for age and sex (table 4). In this model, the odds of all comorbidities were significantly increased in patients designated as having a diagnosis of OSA. Due to the nature of the administrative database, we lacked information on important confounders (i.e. body mass index, race/ethnicity, diet, physical activity and socioeconomic status) and could therefore not adjust for these covariates.

\section{Discussion}

In this very large, nationwide study of health insurance claims for working adults and retirees with employer-sponsored health insurance, we found that the prevalence of important comorbidities is significantly higher in patients with a diagnosis of OSA compared to matched controls. In general, the prevalence of comorbidities increased with ageing in the matched controls, and such trends were ubiquitous among OSA-diagnosed patients as well. However, the increase in prevalence with ageing was amplified by the presence of OSA. Moreover, the joint effects of age and OSA varied based on the comorbidity: the divergence between OSA and controls was more pronounced after the sixth decade of life for most cardiovascular diseases (i.e. heart failure, ischaemic heart disease, stroke and arrhythmias), while depression exhibited an opposite trend. Sex-related differences were also apparent, as hypertension and depression were more prevalent in women with OSA. In contrast, the prevalence of type 2 diabetes and ischaemic heart disease was higher in men with OSA. Notwithstanding the sex and age influences on OSA-imposed morbidity odds, OSA was independently associated with all comorbidities examined in this very large cohort after fully adjusting for confounders. Thus, our findings not only suggest that the risk of comorbidities is increased by OSA but also that this effect also varies by sex and age.

TABLE 3 Specific comorbidities in patients diagnosed with obstructive sleep apnoea (OSA) and matched random samples

\begin{tabular}{lccc} 
& Diagnosed with 0SA & Matched random sample & p-value \\
\hline Total & $1704417(100)$ & $1704905(100)$ & \\
Type 2 diabetes & $236288(13.86)$ & $113640(6.67)$ & $<0.0001$ \\
Hypertension & $299557(17.58)$ & $155851(9.14)$ & $<0.0001$ \\
Arrhythmia & $51389(3.02)$ & $16835(0.99)$ & $<0.0001$ \\
Depression & $92157(5.41)$ & $19456(1.14)$ & $<0.0001$ \\
Ischaemic heart disease & $136584(8.01)$ & $59720(3.50)$ & $<0.0001$ \\
Congestive heart failure & $47002(2.76)$ & $11591(0.68)$ & $<0.0001$ \\
Stroke & $24191(1.42)$ & $7022(0.41)$ & $<0.0001$ \\
Morbid obesity & $55224(3.24)$ & $3802(0.22)$ & $<0.0001$ \\
\hline
\end{tabular}

Data are presented as n (\%) unless otherwise stated. 
The uniqueness of the present study resides in its inclusion of the largest sample of patients with a diagnosis of OSA reported to date, which also incorporates a robust representation of women as well as a wide age spectrum. The large sample size enabled us to explore sex differences and the effect of age in the context of OSA diagnosis and prevalent comorbidities. Our findings are concordant with prior smaller studies reporting a higher prevalence of important comorbidities in patients with OSA [24-26, 29-31]. Such an elevated comorbidity burden has far reaching implications given the increasing prevalence of OSA in the community $[5,6]$.

Before we discuss the implications of our findings, several methodological limitations deserve comment. Our main limitation is inherent to the analysis of a large administrative database. The diagnosis of OSA is based solely on clinicians' use of ICD-9-CM code for billing purposes, such that we cannot exclude the possibility of coding errors or reporting biases. Although we recognise that patients categorised as controls may have
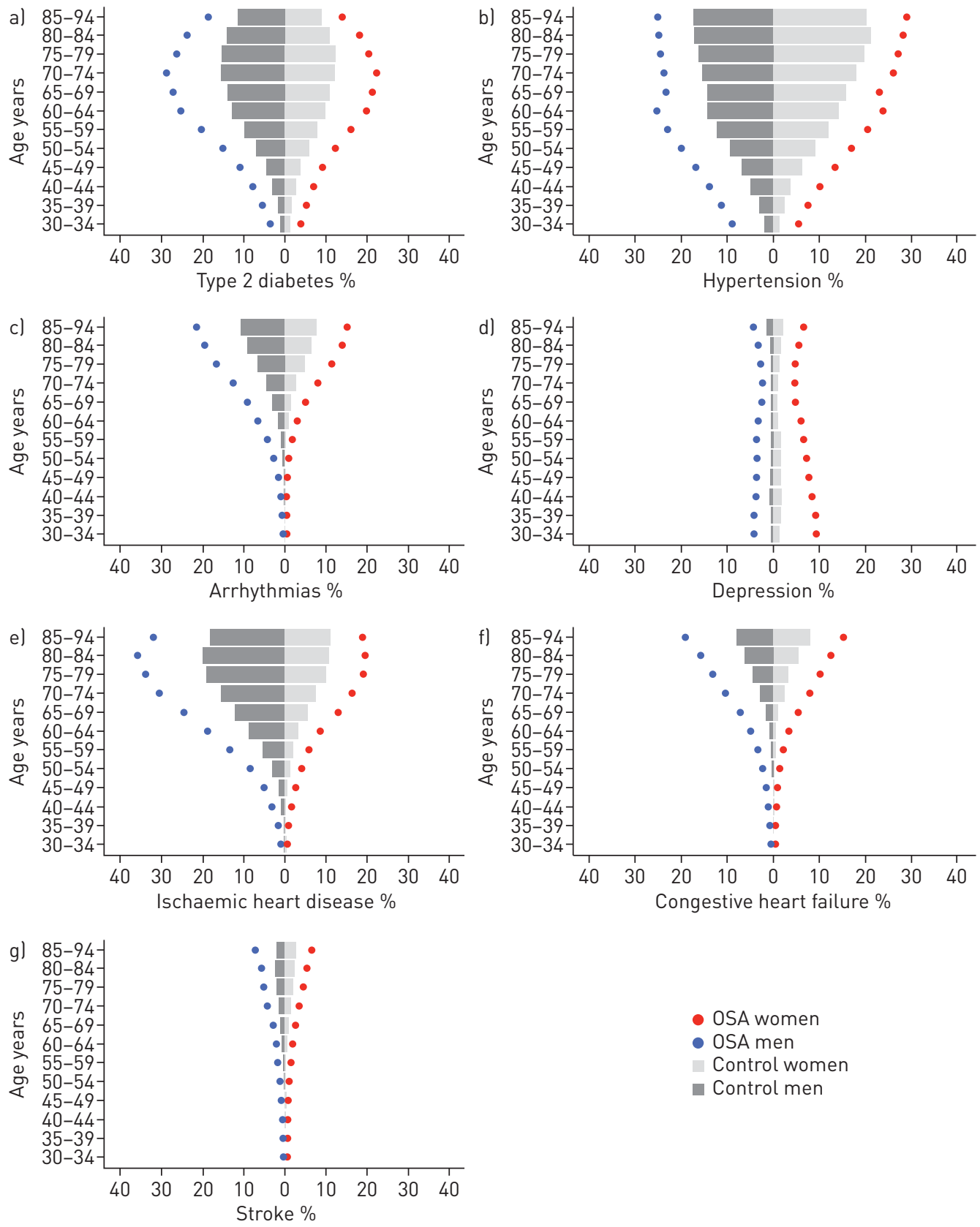

FIGURE 1 Prevalence of a) type 2 diabetes, b) hypertension, c) arrhythmias, d) depression, e) ischaemic heart disease, f) congestive heart failure and gl stroke by age group and sex in patients diagnosed with obstructive sleep apnoea and the matched random sample. 


\begin{tabular}{|c|c|}
\hline Comorbidity & OR $(95 \% \mathrm{CI})$ \\
\hline Type 2 diabetes & $2.29(2.28-2.31)$ \\
\hline Arrhythmia & $3.26(3.20-3.32)$ \\
\hline Ischaemic heart disease & $2.54(2.51-2.56)$ \\
\hline Stroke & $3.51(3.42-3.60)$ \\
\hline Hypertension & $2.14(2.13-2.15)$ \\
\hline Depression & $4.99(4.91-5.07)$ \\
\hline Congestive heart failure & $4.30(4.21-4.39)$ \\
\hline
\end{tabular}

unrecognised or undiagnosed OSA, this would actually serve to bias towards the null, causing our reported effect size to be underestimated. However, it is also possible that the increased prevalence of comorbidities led to increased rates of screening for OSA and, therefore, overestimating the association between OSA and comorbidities. We also minimised the risk of reporting bias (i.e. new diagnosis of OSA leading the clinicians to diagnose additional comorbidities) by querying for diagnosis of comorbidities for the 6 months prior to the index diagnosis of OSA being established. To further exclude the possibility of reporting bias, we also compared the prevalence of a medical condition that is not associated with OSA and/or obesity in both groups. The prevalence of hepatitis $\mathrm{C}$ infection was not different between OSA and controls $(0.11 \%$ versus $0.12 \%$, respectively; $\mathrm{p}=0.24$ ) during the 6 months of the comorbidity assessment. This finding suggests that there was no systematic bias in reporting more comorbidities in patients with OSA.

Low wage and part-time workers were underrepresented in our sample, and the database does not include unemployed individuals. Moreover, the MarketScan database does not include important demographic data that have well-established associations with the prevalence of comorbidities such as body mass index, race/ ethnicity, diet, exercise habits, health literacy and socioeconomic status. This is an important limitation since obesity is a well-recognised risk factor for many of the comorbidities explored in this study as well as a risk factor for OSA. We attempted to partially overcome this limitation by matching for geographical location to the level of state of residence. Nonetheless, we acknowledge that race and obesity are important confounding variables, and their absence constitutes an important limitation, particularly when it applies to our ability to select matched controls. Additional missing information from this national database is the categorical classification of OSA severity, and estimates of adherence to a variety of treatment regimens. Lastly, although our cross-sectional analysis demonstrates a robust association between OSA and prevalent comorbidities, this finding by no means infers causality. However, there is ample rationale to support a biologically plausible link between OSA and increased prevalence of comorbidities, as assessed in our analysis.

Our limitations notwithstanding, this study has several noteworthy strengths. To the best of our knowledge, our sample size is the largest reported thus far, thereby lending more congruency to any of the findings. We purposefully selected equal proportion of men and women with OSA and matched controls to overcome the important limitation of prior studies in which women were significantly underrepresented. This strategy enabled us to perform meaningful comparisons between men and women across various age groups. Accordingly, some of the novel findings indicate that sex has differential effects on the type of comorbidity-OSA interactions, with ischaemic heart disease, for example, being more dominantly represented among male patients, while hypertension and depression show more prominent associations among female patients. The emerging awareness of the prevalence of OSA among women and its potential health-related and sex-related morbid consequences will likely lead to increasing research efforts in this area, such as to tailor more precisely sex-based interventions and outcome measures [33]. Our large sample size also enabled us to better explore the burden of comorbidities according to their distribution patterns among different age groups. When compared to matched controls, the effect of OSA on prevalent cardiovascular comorbidities became apparent around the age of 60-70 years in both sexes, and continued to increase with further ageing. Conversely, the presence of depression showed a different temporal trajectory with aging both in men and in women.

Several studies have shown that patients with OSA will present with increased risk for comorbidities starting several years before a formal diagnosis of OSA is made [25, 27, 28, 30]. This increased burden of comorbidities in patients with OSA leads to significant increase in healthcare resource utilisation, both in younger patients and the elderly [26, 34]. Women with OSA may also be more frequent users of healthcare resources than men [24]. Although the preponderance of evidence to date suggests that there is a high cardiometabolic and neurocognitive comorbidity burden associated with OSA, it remains unclear whether earlier identification and treatment of OSA leads to a reduction in the incidence or severity of 
these comorbidities. To shed light on this challenging and clinically relevant question would require enormous resources such as to perform adequately powered randomised controlled trials. Given the paucity of well-designed clinical trials, analysis of large administrative databases can provide useful real-life data to assist in the design and implementation of future studies [35].

In summary, using a national database that included the largest sample size of men and women with OSA and matched controls to date, the diagnosis of OSA was independently associated with significantly elevated burden of comorbidities. There are important sex differences in the comorbidities explored and the effect of age varies based on the comorbidity. Although early diagnosis and treatment of OSA appears justified due to the multifaceted end-organ morbidity burden imposed by this condition, further well-controlled clinical trials are needed to ascertain whether early identification and treatment of OSA decreases the incidence or severity of comorbidities.

\section{Acknowledgements}

We thank F. Javier Nieto from the University of Wisconsin at Madison (Madison, WI, USA) for helpful discussions.

\section{References}

1 Young T, Palta M, Dempsey J, et al. The occurrence of sleep-disordered breathing among middle-aged adults. N Engl J Med 1993; 328: 1230-1235.

2 Bixler EO, Vgontzas AN, Lin HM, et al. Prevalence of sleep-disordered breathing in women: effects of gender. Am J Respir Crit Care Med 2001; 163: 608-613.

3 Bixler EO, Vgontzas AN, Ten Have T, et al. Effects of age on sleep apnea in men: I. Prevalence and severity. Am J Respir Crit Care Med 1998; 157: 144-148.

4 Duran J, Esnaola S, Rubio R, et al. Obstructive sleep apnea-hypopnea and related clinical features in a population-based sample of subjects aged 30 to 70 yr. Am J Respir Crit Care Med 2001; 163: 685-689.

5 Peppard PE, Young T, Barnet JH, et al. Increased prevalence of sleep-disordered breathing in adults. Am J Epidemiol 2013; 177: 1006-1014.

6 Heinzer R, Vat S, Marques-Vidal P, et al. Prevalence of sleep-disordered breathing in the general population: the HypnoLaus study. Lancet Respir Med 2015; 3: 310-318.

7 Kent BD, Grote L, Ryan S, et al. Diabetes mellitus prevalence and control in sleep-disordered breathing: the European Sleep Apnea Cohort (ESADA) study. Chest 2014; 146: 982-990.

8 Somers VK, White DP, Amin R, et al. Sleep apnea and cardiovascular disease. An American Heart Association/ American College of Cardiology Foundation Scientific Statement from the American Heart Association Council for High Blood Pressure Research Professional Education Committee, Council on Clinical Cardiology, Stroke Council, and Council on Cardiovascular Nursing Council. Circulation 2008; 118: 1080-1111.

9 Young T, Palta M, Dempsey J, et al. Burden of sleep apnea: rationale, design, and major findings of the Wisconsin Sleep Cohort study. WMJ 2009; 108: 246-249.

10 Kohler M, Craig S, Nicoll D, et al. Endothelial function and arterial stiffness in minimally symptomatic obstructive sleep apnea. Am J Respir Crit Care Med 2008; 178: 984-988.

11 Nieto FJ, Peppard PE, Young TB. Sleep disordered breathing and metabolic syndrome. WMJ 2009; 108: 263-265.

12 Buchner NJ, Sanner BM, Borgel J, et al. Continuous positive airway pressure treatment of mild to moderate obstructive sleep apnea reduces cardiovascular risk. Am J Respir Crit Care Med 2007; 176: 1274-1280.

13 Kohler M, Craig S, Pepperell JC, et al. CPAP improves endothelial function in patients with minimally symptomatic OSA: results from a subset study of the MOSAIC trial. Chest 2013; 144: 896-902.

14 Kendzerska T, Gershon AS, Hawker G, et al. Obstructive sleep apnea and incident diabetes. A historical cohort study. Am J Respir Crit Care Med 2014; 190: 218-225.

15 Peppard PE, Young T, Palta M, et al. Prospective study of the association between sleep-disordered breathing and hypertension. N Engl J Med 2000; 342: 1378-1384.

16 Marin JM, Agusti A, Villar I, et al. Association between treated and untreated obstructive sleep apnea and risk of hypertension. JAMA 2012; 307: 2169-2176.

17 Marin JM, Carrizo SJ, Vicente E, et al. Long-term cardiovascular outcomes in men with obstructive sleep apnoea-hypopnoea with or without treatment with continuous positive airway pressure: an observational study. Lancet 2005; 365: 1046-1053.

18 Yaggi HK, Concato J, Kernan WN, et al. Obstructive sleep apnea as a risk factor for stroke and death. $N$ Engl J Med 2005; 353: 2034-2041.

19 Campos-Rodriguez F, Martinez-Garcia MA, de la Cruz-Moron I, et al. Cardiovascular mortality in women with obstructive sleep apnea with or without continuous positive airway pressure treatment: a cohort study. Ann Intern Med 2012; 156: 115-122.

20 Young T, Finn L, Peppard PE, et al. Sleep disordered breathing and mortality: eighteen-year follow-up of the Wisconsin sleep cohort. Sleep 2008; 31: 1071-1078.

21 Punjabi NM, Caffo BS, Goodwin JL, et al. Sleep-disordered breathing and mortality: a prospective cohort study. PLoS Med 2009; 6: e1000132.

22 Peppard PE, Szklo-Coxe M, Hla KM, et al. Longitudinal association of sleep-related breathing disorder and depression. Arch Intern Med 2006; 166: 1709-1715.

23 Leger D, Bayon V, Laaban JP, et al. Impact of sleep apnea on economics. Sleep Med Rev 2012; 16: 455-462.

24 Greenberg-Dotan S, Reuveni H, Simon-Tuval T, et al. Gender differences in morbidity and health care utilization among adult obstructive sleep apnea patients. Sleep 2007; 30: 1173-1180.

25 Banno K, Ramsey C, Walld R, et al. Expenditure on health care in obese women with and without sleep apnea. Sleep 2009; 32: 247-252.

26 Reuveni H, Greenberg-Dotan S, Simon-Tuval T, et al. Elevated healthcare utilisation in young adult males with obstructive sleep apnoea. Eur Respir J 2008; 31: 273-279. 
27 Smith R, Ronald J, Delaive K, et al. What are obstructive sleep apnea patients being treated for prior to this diagnosis? Chest 2002; 121: 164-172.

28 Albarrak M, Banno K, Sabbagh AA, et al. Utilization of healthcare resources in obstructive sleep apnea syndrome: a 5-year follow-up study in men using CPAP. Sleep 2005; 28: 1306-1311.

29 Jennum P, Kjellberg J. Health, social and economical consequences of sleep-disordered breathing: a controlled national study. Thorax 2011; 66: 560-566.

30 Jennum P, Ibsen R, Kjellberg J. Morbidity prior to a diagnosis of sleep-disordered breathing: a controlled national study. J Clin Sleep Med 2013; 9: 103-108.

31 Molnar MZ, Mucsi I, Novak M, et al. Association of incident obstructive sleep apnoea with outcomes in a large cohort of US veterans. Thorax 2015; 70: 888-895.

32 Huang QR, Qin Z, Zhang S, et al. Clinical patterns of obstructive sleep apnea and its comorbid conditions: a data mining approach. J Clin Sleep Med 2008; 4: 543-550.

33 Querejeta Roca G, Redline S, Claggett B, et al. Sex-specific association of sleep apnea severity with subclinical myocardial injury, ventricular hypertrophy, and heart failure risk in a community dwelling cohort: the Atherosclerosis Risk in Communities-Sleep Heart Health Study. Circulation 2015; 132: 1329-1337.

34 Tarasiuk A, Greenberg-Dotan S, Simon-Tuval T, et al. The effect of obstructive sleep apnea on morbidity and health care utilization of middle-aged and older adults. J Am Geriatr Soc 2008; 56: 247-254.

35 Williams JG, Cheung WY, Cohen DR, et al. Can randomised trials rely on existing electronic data? A feasibility study to explore the value of routine data in health technology assessment. Health Technol Assess 2003; 7: 1-117. 